\title{
RIESGOS SOCIONATURALES Y TECNOLÓGICOS EN LAS COMUNIDADES \\ DESDE LA PERCEPCIÓN DE LOS DISCENTES UNIVERSITARIOS
}

José Javier Leal Rivero

(pp.32-56)
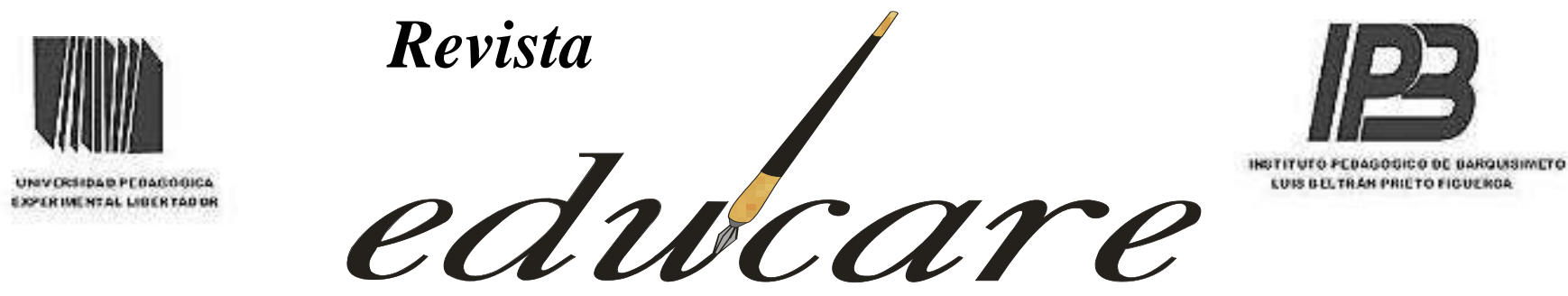

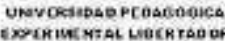

Órgano Divulgativo de la Subdirección de Investigación y Postgrado

del Instituto Pedagógico de Barquisimeto "Luis Beltrán Prieto

Figueroa”

Barquisimeto Estado Lara

Venezuela

Volumen 23 № 3 Septiembre-Diciembre 2019

RIESGOS SOCIONATURALES Y TECNOLÓGICOS EN LAS COMUNIDADES

DESDE LA PERCEPCIÓN DE LOS DISCENTES UNIVERSITARIOS

SOCIONATURAL AND TECHNOLOGICAL RISK IN COMMUNITIES FROM

THE PERCEPTION OF UNIVERSITY DISCIPLES

\section{S S N : $2244-7296$}

\author{
Autor \\ José Javier Leal Rivero \\ Universidad Politécnica Territorial del Estado Trujillo \\ "Mario Briceño Iragorry" (UPTTMBI)
}


RIESGOS SOCIONATURALES Y TECNOLÓGICOS EN LAS COMUNIDADES

DESDE LA PERCEPCIÓN DE LOS DISCENTES UNIVERSITARIOS

José Javier Leal Rivero

\section{Investigación}

Recibido: 03-11-2018

(pp.32-56)

*José Javier Leal Rivero

UPTTMBI

Aceptado: 10-11-2019

\section{ABSTRACT}

\section{RESUMEN}

Se presenta una investigación cuantitativa con diseño de campo exploratoria, con propósito principal de evaluar la percepción de los discentes de la Universidad Politécnica Territorial del estado Trujillo "Mario Briceño Iragorry", respecto a los Riesgos Socionaturales y Tecnológicos que puedan afectar sus comunidades. Se aplicó un cuestionario con respuestas cerradas a setenta (70) estudiantes del Programa Nacional de Formación en Administración. La validez del instrumento se obtuvo mediante un análisis factorial en relación del constructo. La confiabilidad se obtuvo por el Coeficiente Alfa de Cronbach, ubicándose en 0,986. Como resultado se observa que la mayoría de los discentes identifican los riesgos con los desastres que son provocados por eventos asociados a la naturaleza y sus procesos de equilibrio. Se concluye que la percepción es canalizada hacia eventos relacionados con los fenómenos de la naturaleza, limitando el enfoque hacia los aspectos sociales y tecnológicos o las amenazas antrópicas.

Descriptores: Riesgos socionaturales; riesgos tecnológicos; comunidades.
Quantitative research is presented with exploratory field design, with the main purpose of assessing the perception of the speakers of the Territorial Polytechnic University of the state Trujillo "Mario Briceño Iragorry", regarding the Risks Socionatural and Technological that may affect their communities. A questionnaire was applied with closed responses to seventy (70) students of the National Management Training Program. The validity of the instrument was obtained by a factoranalysis in relation to the construct. Reliability was obtained by Cronbach's Alpha Coefficient, which stood at 0.986 . As a result, most speakers identify risks with disasters that are caused by events associated with nature and its balancing processes. It is concluded that perception is channeled into events related to the phenomena of nature, limiting the focus on social and technological aspects or anthropic threats.

Key words: Socionatural risks; technological risks; communities

*Profesor Asociado Universidad Politécnica Territorial del Estado Trujillo "Mario Briceño Iragorry" (UPTTMBI) - Venezuela. Ing. Industrial-UVM. Magister Scientiarum en Gestión de Políticas Públicas-UBV y Doctorante en Ciencias de la Educación Universidad Nacional Experimental "Simón Rodríguez"(UNESR). liderunox@gmail.com

Volumen 23, $N^{\circ} 3$, Septiembre-Diciembre 2019. ISSN 2244-7296 


\section{RIESGOS SOCIONATURALES Y TECNOLÓGICOS EN LAS COMUNIDADES \\ DESDE LA PERCEPCIÓN DE LOS DISCENTES UNIVERSITARIOS \\ José Javier Leal Rivero}

(pp.32-56)

\section{Introducción}

\section{Contextualización de la temática}

A pesar del avance tecnológico y comunicacional que vive la humanidad, hay aspectos en los que parece estancarse, o no decidirse a superar, como es el caso de algunos paradigmas que no le permiten conformar una sociedad más sustentable y armoniosa con la naturaleza. Así, puede observarse como a lo largo de la historia, la humanidad no ha dejado de enfrentarse al terrible hostigamiento de las fuerzas vivas de la naturaleza, las cuales realmente siempre están buscando su equilibrio, pero que el hombre, desde la antigüedad y todavía en algunas sociedades, en su ignorancia que conlleva el pensamiento mágico, produce en las personas una resignación fatalista a estos eventos, acompañado de un sentimiento de impotencia y pasividad.

Así, puede observarse que países como Venezuela y Brasil, se encuentran entre aquellos con el mayor número de personas afectadas en el mundo para los últimos veinte años, por desastres naturales relacionados con el clima, según el estudio publicado por la Oficina de las Naciones Unidas para la Reducción del Riesgo de Desastres, UNISDR (2015).

Más alarmantemente, se señala en el citado informe que, en función del número de fallecidos a causa de este tipo de desastres en proporción al número de habitantes, Venezuela se sitúa como el segundo país más afectado entre 1995 y 2015, por debajo de Myanmar, con 126 muertos por cada millón de habitantes, mientras que Brasil ocupa el séptimo lugar en el listado de países con el mayor número de personas afectadas a nivel mundial en ese mismo período, con 51 millones de perjudicados (op.cit).

Del total de los 157.000 fallecidos, a nivel mundial entre 1995 y 2015 por inundaciones, 30.239 personas perecieron en Venezuela, casi la totalidad en las inundaciones registradas en el estado de Vargas (el mayor desastre conocido en la historia venezolana), Miranda y Falcón en 1999. Relacionándose esa situación, con los datos del Centro de Investigación sobre la Epidemiología de los Desastres (CRED) en los que se apoya el informe de la ONU

Volumen 23, $N^{\circ} 3$, Septiembre-Diciembre 2019. ISSN 2244-7296 
RIESGOS SOCIONATURALES Y TECNOLÓGICOS EN LAS COMUNIDADES

DESDE LA PERCEPCIÓN DE LOS DISCENTES UNIVERSITARIOS

José Javier Leal Rivero

(pp.32-56)

(op.cit), los cuales apuntan al hecho que los desastres meteorológicos con mayor incidencia observados en Venezuela, son las inundaciones.

No obstante, ya se reconoce que en la medida en que los sujetos conocen el riesgo que representan estos fenómenos naturales, perciben sus propios peligros internos y aprenden a enfrentar ambos tipos de amenazas, podrán atenuar los efectos de su impacto, reduciendo la probabilidad de que se transformen en auténticos desastres.

En ese contexto, la gestión integral del riesgo de desastre, aplicada según la normativa venezolana, Ley de Gestión Integral de Riesgos Socionaturales y Tecnológicos, LGIRSyT, (2009), se ha convertido en un tema de gran importancia debido al incremento de los desastres producidos en los últimos años, lo que ha provocado consigo un aumento en pérdidas humanas, económicas y materiales. En consecuencia, la tendencia positiva se debe centrar en los esfuerzos para la reducción de riesgos y vulnerabilidades que proporcionen una solución satisfactoria a todos los agentes implicados en un desastre, desde el gobierno y las instituciones, entre otros, hasta lo más importante que son las personas afectadas y que en la mayoría de los casos son las que tienen menor información y desconocen los riesgos a los que pueden enfrentarse.

Por esa razón, y aprovechando la transversalidad que permiten los nuevos Programas Nacionales de Formación, creados por iniciativa del Estado venezolano, se genera la iniciativa del presente estudio, el cual se propuso como propósito principal identificar la percepción que poseen los discentes de la Universidad Politécnica Territorial del estado Trujillo "Mario Briceño Iragorry” (UPTTMBI), en relación a los Riesgos Socionaturales y Tecnológicos que puedan afectar sus comunidades.

El mismo, se enfocó dentro del paradigma cuantitativo, conformando parte de una investigación más profunda realizada en el contexto del desarrollo de estrategias para el fortalecimiento de la cultura de promoción y aprendizaje en Gestión Integral de Riesgos Socionaturales y Tecnológicos en los discentes de la UPTTMBI (Leal, 2016), buscando, a través de este la atención de un proceso científico exploratorio, que permitieran facilitar

Volumen 23, $N^{\circ} 3$, Septiembre-Diciembre 2019. ISSN 2244-7296 


\section{RIESGOS SOCIONATURALES Y TECNOLÓGICOS EN LAS COMUNIDADES}

DESDE LA PERCEPCIÓN DE LOS DISCENTES UNIVERSITARIOS

José Javier Leal Rivero

(pp.32-56)

hallazgos generadores de estrategias didácticas novedosas, en el ámbito de las Universidades venezolanas.

\section{Argumentación Teórica}

Comúnmente, se señala que el riesgo es una situación que puede conducir a una consecuencia negativa no deseada en un acontecimiento, o bien es la probabilidad de que suceda un determinado peligro potencial, entendiéndolo como una situación física que puede provocar daños a la vida, a los equipos o al medio ambiente. También, es asociarlo a las consecuencias no deseadas de una actividad dada, en relación con la probabilidad de que ocurra.

Autores como Perry (2003), ofrecen otro punto de vista sobre el riesgo como una medida de la pérdida económica o del daño a las personas tanto en probabilidad del incidente como en la magnitud del daño o injuria. Mientras que la Estrategia Internacional para la Reducción de los Desastres, (EIRD) (2004), lo definió como la probabilidad de consecuencias perjudiciales o pérdidas esperadas (muertes, lesiones, propiedad, medios de subsistencia, interrupción de actividad económica o deterioro ambiental) resultado de interacciones entre amenazas naturales o antropogénicas y condiciones de vulnerabilidad.

En el ámbito normativo venezolano, el Articulo 5, numeral 11 de Ley de Gestión Integral de Riesgos Socionaturales y Tecnológicos, LGIRSyT, (2009), señala que "Riesgo construido. Son aquellas condiciones generadas por el Estado, el sector privado o la sociedad en general que pudieran causar o potenciar desastres de carácter socionatural o tecnológico". Por ello, se puede establecer que los riesgos, que pueden conllevar a desastres, son eminentemente ocasionados por la acción del hombre, una consecuencia directa de sus actos.

\section{Los Desastres}

Definir el termino desastre, parece depender de la disciplina donde sea utilizado el mismo, esto debido a la inexistencia de una definición universalmente aceptada, según lo

Volumen 23, $N^{\circ} 3$, Septiembre-Diciembre 2019. ISSN 2244-7296 
RIESGOS SOCIONATURALES Y TECNOLÓGICOS EN LAS COMUNIDADES

DESDE LA PERCEPCIÓN DE LOS DISCENTES UNIVERSITARIOS

José Javier Leal Rivero

(pp.32-56)

refieren Shaluf y Fakhrul-Razi (2013). Así, muchas veces, los términos desastre y catástrofe se usan de manera indistinta aunque difieren en su raíz etimológica.

El desastre, definido en términos sociales, físicos y sanitarios, se torna a su vez en catástrofe subjetiva. Donde la catástrofe puede ser representada como una combinación desbalanceada de procesos naturales, tecnológicos, organizacionales, mentales y físicos, que resultan de una incorrecta representación de dichos procesos, mala explicación de la información que se tiene acerca de ellos, conocimiento incorrecto de la gestión y su falta de predicción. (Perry y Quarantelli, 2004)

El desastre también, también puede ser definido como un evento inusual natural o creado por el hombre, que incluye eventos causados por fallas de sistemas tecnológicos, que temporalmente destruyen la capacidad de respuesta de sociedades humanas, grupos o individuos y medio ambiente, sus causas son grandes daños masivos, pérdidas económicas, disrupciones, y en ocasiones perdida de la vida (Shalufet et al., 2013).

Los desastres a diferencia de las emergencias, son impactos peligrosos a la comunidad, causan problemas únicos a las organizaciones públicas y privadas, afectando también a los gobiernos locales, estatales y nacionales. Los desastres tienen la característica de dañar a las personas, a la propiedad y al ambiente (Mansor, 2004). En cambio, las emergencias de rutina y los incidentes de crisis son eventos que no exceden la capacidad normal de cualquier organización o gobierno. Estos eventos pueden incluir accidentes de vehículos de motor, suicidios, fuegos y tiroteos.

En el caso venezolano, se atiende a la definición que refiere el Artículo 5, numeral 2 de la LGIRSyT(2009), en el cual se señala, que los desastres son, “Alteraciones graves en las personas, los bienes, los servicios y el ambiente, causadas por un suceso natural o generado por la actividad humana, que exceden la capacidad de respuesta de la comunidad afectada".

\section{Riesgos Socionaturales}

La Ley define los riesgos socionaturales como el "peligro potencial asociado con la probable ocurrencia de fenómenos físicos cuya existencia, intensidad o recurrencia se

Volumen 23, $N^{\circ} 3$, Septiembre-Diciembre 2019. ISSN 2244-7296 
RIESGOS SOCIONATURALES Y TECNOLÓGICOS EN LAS COMUNIDADES

DESDE LA PERCEPCIÓN DE LOS DISCENTES UNIVERSITARIOS

José Javier Leal Rivero

(pp.32-56)

relaciona con procesos de degradación ambiental o de intervención humana en los ecosistemas naturales" (Artículo 5, num. 12).

La citada disposición contempla dos supuestos: (1) procesos de degradación ambiental y; (2) acciones de intervención humana directa en ecosistemas naturales. Este último supuesto es lo suficientemente explícito y no presenta dudas sobre su alcance, puesto que siempre se refiere a riesgos o daños derivados de la acción humana directa sobre el ambiente.

En lo que respecta al primer supuesto, se deja entendido que el término "degradación ambiental", evidencia que el proceso con efectos en la naturaleza es causado por un elemento externo que "degrada" el ambiente, siendo este el factor humano. La degradación implica, por tanto, un elemento externo que degrada a la cosa, por lo que no es un término aplicado al ambiente para hacer referencia a fenómenos exclusivamente naturales.

De ese modo, la degradación ambiental ha sido definida, para efectos de la Ley, como el conjunto de procesos que deterioran o impiden la utilización de un determinado recurso, tal como el agua, el suelo fértil, el paisaje, entre otros, por parte de la Humanidad. De esta forma los procesos de degradación ambiental son entendidos como procesos socioeconómicos en tanto que imposibilitan la adecuada utilización de un recurso determinado (Artieda y Palacios, 2000).

Así, la degradación ambiental no puede ser catalogada como un fenómeno físico de la naturaleza que opera por sí solo, sino como una consecuencia de las acciones que los humanos tienen sobre la naturaleza, que pueden desencadenar riesgos o daños directos. Se percibe, en ese sentido, que el término usado para la degradación autónoma por causas químicas es biodegradación, el cual contrasta con el de degradación ambiental.

Lo anterior se ratifica en la medida en que la Ley utiliza el novedoso término "riesgos socionaturales", que implica un factor social (humano), en lugar hacer mención a riesgos exclusivamente naturales, los cuales se identificarían con desastres, catástrofes o calamidades y no con la intervención del hombre.

Volumen 23, $N^{\circ} 3$, Septiembre-Diciembre 2019. ISSN 2244-7296 


\section{RIESGOS SOCIONATURALES Y TECNOLÓGICOS EN LAS COMUNIDADES \\ DESDE LA PERCEPCIÓN DE LOS DISCENTES UNIVERSITARIOS \\ José Javier Leal Rivero \\ (pp.32-56)}

\section{Riesgos Tecnológicos}

La Ley define, de una manera directa y precisa, los riesgos tecnológicos como “el peligro potencial generado por la actividad humana relacionado con el acceso o uso de la tecnología, percibidos como eventos controlables por el hombre o que son fruto de su actividad" (Articulo. 5, núm. 13).

A continuación se presenta, a modo de resumen, un cuadro sinóptico que permite observar con mayor claridad la clasificación de los riesgos, tal como son definidos por la normativa venezolana.

Cuadro 1. Sinóptico clasificación de riesgos por tipo

\begin{tabular}{|c|c|}
\hline Naturales & Antrópicos \\
\hline Hidrometeorologicos & Sociales \\
\hline - Tormentas tropicales & - Accidentes \\
\hline - Precipitaciones de graniza & terrestre, aéreos, acuáticos. \\
\hline - Huracanes o ciclones & Incendios de estructuras. \\
\hline - Tornados & Conmoción social. \\
\hline - Olas de frio & Terrorismo. \\
\hline - Olas de calor o sequias & - Beligerancia. \\
\hline Geológicos & Tecnológicos \\
\hline - Terremotos (sismos). & - Materiales peligrosos. \\
\hline Maremotos (tsunami). & - Petroleros \\
\hline Astronómicos & Gasiferos. \\
\hline $\begin{array}{l}\text { - Impactos por meteoros o } \\
\text { masas similares. }\end{array}$ & $\begin{array}{l}\text { - Termo e hidroeléctricos. } \\
\text { _ Industrial. }\end{array}$ \\
\hline Hidrogeomorfológicos & \\
\hline - Deslizamientos. & \\
\hline $\begin{array}{l}\text { Derrumbes. } \\
\text { Inundaciones. }\end{array}$ & \\
\hline
\end{tabular}

Nota: Interpretación del Autor, 2016.

\section{La Vulnerabilidad}

El EIRD (2004), definió la vulnerabilidad como las condiciones determinadas por factores o procesos físicos, sociales, económicos y ambientales, que aumentan la susceptibilidad de una comunidad al impacto de amenazas. Autores como Cardona (2003, p.67), puntualizan el concepto vulnerabilidad, en el contexto de la gestión del riesgo, como:

El factor de riesgo interno de un elemento o grupo de elementos expuestos a una amenaza, correspondiente a su predisposición intrínseca o susceptibilidad física, económica, social y política que tiene una comunidad de ser afectada o de sufrir

Volumen 23, $N^{\circ} 3$, Septiembre-Diciembre 2019. ISSN 2244-7296 
RIESGOS SOCIONATURALES Y TECNOLÓGICOS EN LAS COMUNIDADES

DESDE LA PERCEPCIÓN DE LOS DISCENTES UNIVERSITARIOS

José Javier Leal Rivero

(pp.32-56)

efectos adversos en caso de que se manifieste un fenómeno peligroso de origen natural, socio natural o antropogénico.

Simplificándolo, como un factor de riesgo interno, se puede formular como "la probabilidad de que el sistema, o el sujeto expuesto, sean afectados por el suceso o fenómeno que caracteriza la amenaza" (Cardona, 2007, p.146). Por otro lado, Myers (2004), expresa que la vulnerabilidad es la susceptibilidad ante la pérdida o daño, provocada por una amenaza.

Una vez más, enfocando la definición al contexto nacional, se puede observar en la LGIRSyT, específicamente en el artículo 5, numeral 14 que, la vulnerabilidad son, "Condiciones inadecuadas de seguridad que presentan personas, edificaciones, espacios físicos, entre otros, ante una amenaza potencialmente dañina”.

Es necesario acotar, que la vulnerabilidad, representa, además una falta de resiliencia para recuperase posteriormente. Las diferencias de vulnerabilidad del contexto social y material expuesto ante un fenómeno peligroso determinan el carácter selectivo de la severidad de sus efectos. Así, el reconocer que la vulnerabilidad es un elemento clave en la formulación del riesgo, incrementa el interés por vincular las capacidades de las personas para hacer frente a los efectos de las amenazas.

\section{Dimensiones de la Vulnerabilidad}

Dependiendo de los diferentes aspectos, o enfoques, que caracterizan la vulnerabilidad, esta puede tener varias dimensiones, las cuales, según Cardona (2007) y el EIRD (2004), podrían resumirse, de la siguiente forma:

a) Dimensión física. Este concepto tiene connotación material, proviene de las corrientes de ordenamiento territorial, de la ingeniería y la arquitectura. Aquí se expresan las características de ubicación en áreas propensas y las deficiencias de resistencia de los elementos expuestos, de los que depende su capacidad de absorber la acción del suceso que representa la amenaza.

Volumen 23, $N^{\circ} 3$, Septiembre-Diciembre 2019. ISSN 2244-7296 
RIESGOS SOCIONATURALES Y TECNOLÓGICOS EN LAS COMUNIDADES

DESDE LA PERCEPCIÓN DE LOS DISCENTES UNIVERSITARIOS

José Javier Leal Rivero

(pp.32-56)

b) Dimensión económica. El grado de vulnerabilidad depende mucho de la situación económica de las personas, comunidades y países. La población pobre, grupo que en la mayoría de las regiones está formado por una excesiva proporción de mujeres y ancianos, generalmente son mucho más vulnerables que los segmentos más acomodados de la sociedad. Al nivel local e individual este aspecto se expresa en desempleo, insuficiencia de ingresos, dificultad o imposibilidad de acceso a los servicios. En la esfera nacional se traduce en una excesiva dependencia económica de factores externos incontrolables, la falta de diversificación de la base económica, las restricciones al comercio internacional y la imposición de políticas monetarias.

c) Dimensión social. Esta guarda relación con el grado de bienestar de las personas, las comunidades y la sociedad. Cuanto más esté integrada una comunidad, mejor puede superar los inconvenientes que suelen presentarse, resultará más fácil absorber las consecuencias de un desastre y podrá reaccionar con mayor rapidez que una comunidad que no lo esté.

d) Dimensión educativa. La ausencia de conocimiento sobre las causas, los efectos y las razones por las cuales se presentan desastres, el desconocimiento de la historia, la falta de preparación y desconocimiento del comportamiento individual y colectivo, en caso de desastre son aspectos que hacen que una comunidad sea más vulnerable. Una educación deficiente o que no tiene buena cobertura es una comunidad propensa y la falta de socialización de la información aumentan la vulnerabilidad.

e) Dimensión política. Se expresa en el nivel de autonomía que tiene una comunidad con respecto a sus recursos y por la toma de decisiones que la afectan. La comunidad se hace más vulnerable bajo esquemas centralistas en la toma de decisiones y en la organización gubernamental. La debilidad en los niveles de autonomía a los problemas sentidos en estos niveles territoriales. En la medida que la comunidad participa más en las decisiones que le atañen es menos vulnerable.

f) Dimensión institucional. Está relacionada con las dificultades que tienen las instituciones para hacer la gestión del riesgo. Situación que se refleja en la falta de

Volumen 23, $N^{\circ} 3$, Septiembre-Diciembre 2019. ISSN 2244-7296 
RIESGOS SOCIONATURALES Y TECNOLÓGICOS EN LAS COMUNIDADES

DESDE LA PERCEPCIÓN DE LOS DISCENTES UNIVERSITARIOS

José Javier Leal Rivero

(pp.32-56)

preparación para responder ante un suceso, o cuando aun sabiendo que existe el riesgo no llevan a cabo acciones eficientes y efectivas para reducirlo o mitigarlo. Se expresa en la falta de flexibilidad de las instituciones, en el exceso de burocracia, en el hecho de que prevalecen la decisión política y el protagonismo.

g) Dimensión cultural. Esta dimensión de la vulnerabilidad está referida a la forma en que los individuos se ven a sí mismos en la sociedad y como colectividad, lo que influye en ocasiones de manera negativa debido a estereotipos perniciosos que no se cuestionan y que se consolidan. Al respecto, juegan un papel crucial los medios de comunicación, puesto que contribuyen a la utilización sesgada de imágenes o a la transmisión de información ligera o imprecisa sobre el medio ambiente, la misma sociedad y los desastres.

h) Dimensión ambiental. En esta dimensión, cabe mencionar el grado de agotamiento de los recursos naturales y su estado de degradación. Del mismo modo, la falta de resiliencia de los sistemas ecológicos y la exposición a contaminantes tóxicos y peligrosos son factores importantes que contribuyen a la vulnerabilidad ambiental. Estas circunstancias conducen al deterioro de los ecosistemas y a aumentar la vulnerabilidad debido a la incapacidad de auto ajustarse para compensar los efectos directos o indirectos de la acción humana o de sucesos de la misma naturaleza.

i) Dimensión ideológica. Está relacionada con las ideas o creencias que tiene las personas sobre el devenir y los hechos del mundo. Se expresa en actitudes pasivas, fatalistas y creencias religiosas que limitan la capacidad de actuar de los individuos en ciertas circunstancias. La percepción dogmática de las cosas puede generar confusión acerca de un propósito, falta de reacción y muchas veces pérdida de la motivación, que debilitan una acción transformadora.

Desde el punto de vista de la gestión del riesgo, las acciones que reduzcan los factores negativos, dentro de cada dimensión señalada anteriormente, y que estimulen las fortalezas y capacidades de una comunidad, entendida como los elementos expuestos, deben ser el objetivo de la planificación y la prevención-mitigación.

Volumen 23, $N^{\circ} 3$, Septiembre-Diciembre 2019. ISSN 2244-7296 
RIESGOS SOCIONATURALES Y TECNOLÓGICOS EN LAS COMUNIDADES

DESDE LA PERCEPCIÓN DE LOS DISCENTES UNIVERSITARIOS

José Javier Leal Rivero

(pp.32-56)

\section{La Amenaza}

La amenaza, como elemento constituyente del riesgo, normalmente hace referencia a lo que diversos autores denominan como peligro o peligrosidad. Ambos conceptos pueden considerarse como sinónimos para efectos de un enfoque conceptual. Al respecto, Perry (2003), señala que el peligro es definido como, el potencial inherente de una sustancia o actividad para dañar a las personas, a los bienes o al medio ambiente. Mansor (2004), por su parte, refiere que la amenaza es, una parte inevitable de la vida. La amenaza es el potencial, mientras que el desastre es el acontecimiento real.

Para Cardona (2007), desde el punto de vista conceptual, la amenaza se debe entender como un factor de riesgo externo, de un elemento o grupo de elementos expuestos, que se expresa como la probabilidad de que un suceso se presente con cierta intensidad, en un sitio especifico y dentro de un periodo de tiempo definido. Mientras que en el ámbito de la legislación venezolana, la LGIRSyT (2009), específicamente en el artículo 5, numeral 1, se define la amenaza como la:

Probabilidad de que un fenómeno se presente con una cierta intensidad, en un sitio específico y dentro de un período de tiempo definido, con potencial de producir efectos adversos sobre las personas, los bienes, los servicios y el ambiente.

Es necesario resaltar en este punto, que los fenómenos o eventos naturales erróneamente han sido denominados "desastres naturales", y son clasificados de diferente manera, dependiendo del contexto en el que se utilicen. Cabe destacar, entonces que, el fenómeno natural únicamente se convierte en amenaza cuando hay personas y bienes que pueden ser afectados, posteriormente convertirse en un desastre cuando esta amenaza impacta en las personas y sus recursos son destruidos.

\section{Clasificación de las Amenazas en relación a su origen}

Como puede comprenderse, existe una amplia gama de amenazas, que en ocasiones ocurren por sí solas o mediante complejas formas de interacción, las cuales pueden poner en peligro la vida de las personas y el desarrollo sostenible, causando daños enormes, 


\section{RIESGOS SOCIONATURALES Y TECNOLÓGICOS EN LAS COMUNIDADES}

DESDE LA PERCEPCIÓN DE LOS DISCENTES UNIVERSITARIOS

José Javier Leal Rivero

(pp.32-56)

provocando lesiones, contaminación o muerte (Mansor, 2004). Si se reconsidera su origen, las amenazas pueden clasificarse en tres grupos:

Las causadas por fenómenos naturales, que tienen su origen en los procesos de transformación y la dinámica natural del planeta Tierra. A su vez estas pueden catalogarse en:

a) Geológicas: sismos, erupciones volcánicas, maremotos (tsunamis), deslizamientos, derrumbes, erosión, entre otros.

b) Hidrometeorológicas: huracanes, tormentas tropicales, tormentas eléctricas, sequías, fenómeno de El Niño, temperaturas extremas, inundaciones.

Las que obedecen a causas tecnológicas o antropogénicas, también denominadas antrópicas, ocasionados directamente por la acción del hombre, entre las que se encuentran, las explosiones, incendios, accidentes de tránsito o aéreos en zonas urbanas, fugas radiológicas, contaminación del aire, agua o suelo, entre muchas otras.

Y por último en, amenazas Sociales: relacionadas con conflictos sociales, tales como disturbios sociales, guerras, personales, entre otros.

Puede considerarse también, la posibilidad que se produzcan combinaciones, respecto al origen y tipo de amenazas, lo que daría pie a situaciones de conformación de Amenazas Complejas, o Concatenadas, esto podría darse al ocurrir un evento físico determinado que desencadene una serie de otros eventos. Por ejemplo, un terremoto puede detonar derrumbes, incendios, ruptura de oleoductos o presas de agua causando inundaciones; un huracán se asocia con inundaciones, deslizamientos, destrucción de almacenes de materiales peligrosos y otros.

Del mismo modo, se presentan los Contextos de Multiamenaza, los cuales se pueden relacionar con situaciones diversas, por ejemplo, una zona particular puede ser afectada en momentos distintos, o a la vez, por distintos tipos de evento: inundaciones, deslizamientos, erosión, sismos, incidentes en las instalaciones industriales producto de la presencia de algún evento natural o antrópico.

Volumen 23, $N^{\circ} 3$, Septiembre-Diciembre 2019. ISSN 2244-7296 
RIESGOS SOCIONATURALES Y TECNOLÓGICOS EN LAS COMUNIDADES

DESDE LA PERCEPCIÓN DE LOS DISCENTES UNIVERSITARIOS

José Javier Leal Rivero

(pp.32-56)

Un caso emblemático de estas dos últimas consideraciones clasificatorias, se evidenció recientemente con el terremoto y tsunami de Japón de 2011, denominado oficialmente por la Agencia Meteorológica de Japón como el terremoto de la costa del Pacífico en la región de Tōhoku de 2011, fue un terremoto de magnitud 9,0 MW que creó olas de maremoto de hasta 10 mts. Posteriormente, a causa de estos eventos, se declaró un estado de emergencia en la central nuclear de Fukushima 1 y 2 de la empresa Tokyo Electric Power (TEPCO), a causa de la falla de los sistemas de refrigeración de uno de los reactores, por lo que en un principio se habían evacuado a los 3000 pobladores en un radio de $3 \mathrm{~km}$ del reactor.

Posteriormente las autoridades Japonesas, se vieron en la obligación de dar una categoría de 4 en una escala de 7 en la Escala Internacional de Accidentes Nucleares evacuando a más 45000 personas y comenzando a distribuir yodo, elemento eficaz en contra el Cáncer de tiroides derivado de la peligrosa Radiación nuclear. Se calificó este incidente como el más grave desde el accidente de Chernóbil.

Ahora bien, casi todas las comunidades, sean urbanas o rurales, son vulnerables a las amenazas, siendo las más comunes las hidrometeorológicas, teniéndose las inundaciones, como las que afectan a dos tercios de la población afectada por amenazas de origen natural, esto según datos del EIRD (2004), siendo estos datos, a su vez, consistentes con los eventos catastróficos presentados anteriormente, para el caso de Venezuela.

\section{Relación entre Riesgo, Vulnerabilidad, Exposición y Amenaza}

Una vez finalizadas las definiciones básicas, relacionadas con el riesgo, puede inferirse, que éste, también señalado como riesgo de desastre, se fundamenta en la relación existente, entre la vulnerabilidad, la exposición y la amenaza.

Esa relación puede representarse de la siguiente forma:

Volumen 23, $N^{\circ} 3$, Septiembre-Diciembre 2019. ISSN 2244-7296 


\section{RIESGOS SOCIONATURALES Y TECNOLÓGICOS EN LAS COMUNIDADES \\ DESDE LA PERCEPCIÓN DE LOS DISCENTES UNIVERSITARIOS \\ José Javier Leal Rivero}

(pp.32-56)

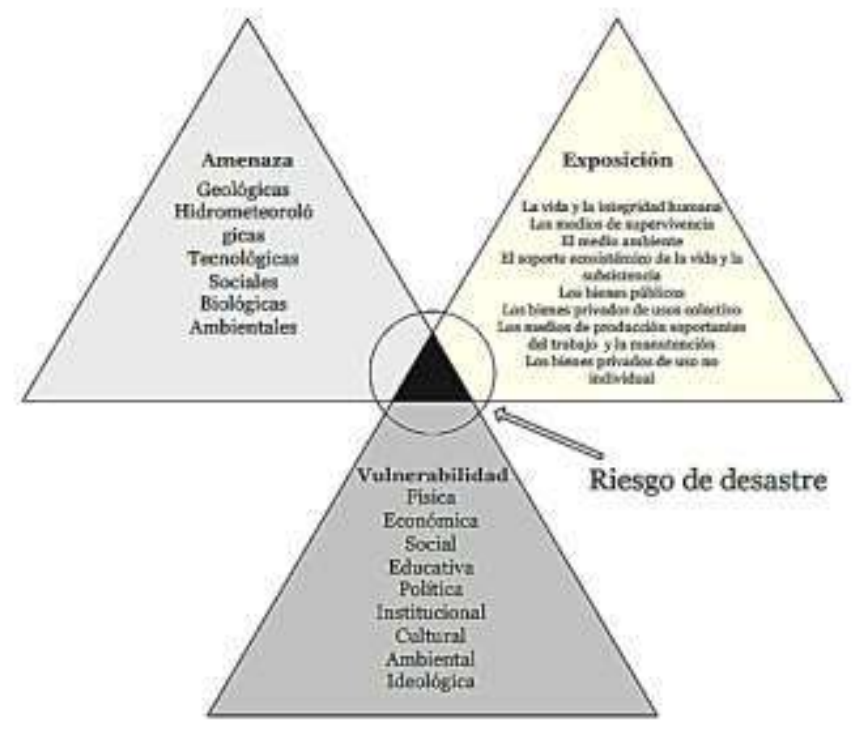

Gráfico 1.Aproximación a la Relación entre Riesgo, Vulnerabilidad, Exposición y Amenaza. Fuente: Carreño, 2007

Finalizado el aporte conceptual, y entendiendo la diversidad de puntos de vista existentes con referencia a los mismos, se precisa señalar la forma en que se unificaron los diferentes criterios, para así hacerlos más manejables y estandarizados a nivel mundial, permitiendo manejar una terminología común, independientemente del país en el que se desarrollen las acciones referentes a la Gestión de Riesgos, y la manera para modelar o formular matemáticamente, la relación existente entre los elementos hasta ahora señalados.

Así, la Oficina de las Naciones Unidas para la Mitigación de Desastres, UNDRO (actualmente Oficina de las Naciones Unidas para la Reducción del Riesgo de Desastres, UNISDR) y la Organización de las Naciones Unidas para la Educación, la Ciencia y la Cultura, UNESCO, promovieron una reunión de expertos con el fin de proponer una unificación de definiciones. El informe de dicha reunión, llamado Natural Disasters and Vulnerability Analysis (UNDRO 1979) incluyó las siguientes definiciones (Cardona, 2001, pp.08-10):

a) Amenaza, peligro o peligrosidad (Hazard - H). Es la probabilidad de ocurrencia de un suceso potencialmente desastroso durante cierto período de tiempo en un sitio dado.

Volumen 23, $N^{\circ} 3$, Septiembre-Diciembre 2019. ISSN 2244-7296 
RIESGOS SOCIONATURALES Y TECNOLÓGICOS EN LAS COMUNIDADES

DESDE LA PERCEPCIÓN DE LOS DISCENTES UNIVERSITARIOS

José Javier Leal Rivero

(pp.32-56)

b) Vulnerabilidad (Vulnerability -V). Es el grado de pérdida de un elemento o grupo de elementos bajo riesgo resultado de la probable ocurrencia de un suceso desastroso, expresada en una escala desde 0 o sin daño a 1 o pérdida total.

c) Riesgo específico (Specific Risk - Rs). Es el grado de pérdidas esperadas debido a la ocurrencia de un suceso particular y como una función de la amenaza y la vulnerabilidad.

d) Elementos en riesgo (Elements at Risk - E). Son la población, los edificios y obras civiles, las actividades económicas, los servicios públicos, las utilidades y la infraestructura expuesta en un área determinada.

e) Riesgo total (Total Risk - Rt). Se define como el número de pérdidas humanas, heridos, daños a las propiedades y efectos sobre la actividad económica debido a la ocurrencia de un desastre, es decir el producto del riesgo específico Rs, y los elementos en riesgo E. De esta manera, desde ese punto de vista, la evaluación del riesgo puede llevarse a cabo mediante la siguiente formulación general (ibidem):

$$
\mathbf{R t}=\mathbf{E} \cdot \mathbf{R s}=\mathbf{E} \cdot \mathbf{H} \cdot \mathbf{V}
$$

Esta manera de

conceptuar la amenaza, la vulnerabilidad y el riesgo, ampliamente aceptada desde entonces en el campo técnico y científico, y con mayor frecuencia utilizada en las ciencias sociales, fue planteada por el autor antes citado de la siguiente manera (ibídem):

$$
\text { Rie }|\mathbf{t}=(\mathrm{Ai}, \mathrm{Ve})| \mathbf{t}
$$

En otras palabras, esto significa que una vez conocida la amenaza o peligro "Ai", entendida como la probabilidad de que se presente un suceso con una intensidad mayor o igual a "i" durante un período de exposición " $\mathrm{t}$ ", y conocida la vulnerabilidad "Ve", entendida como la predisposición intrínseca de un elemento expuesto e a ser afectado o de ser susceptible a sufrir un daño ante la ocurrencia de un suceso con una intensidad "i”, el riesgo "Rie" se expresa como la probabilidad de que se presente una pérdida sobre el

Volumen 23, $N^{\circ} 3$, Septiembre-Diciembre 2019. ISSN 2244-7296 
RIESGOS SOCIONATURALES Y TECNOLÓGICOS EN LAS COMUNIDADES

DESDE LA PERCEPCIÓN DE LOS DISCENTES UNIVERSITARIOS

José Javier Leal Rivero

(pp.32-56)

elemento "e", como resultado de la ocurrencia de un suceso con una intensidad mayor o igual a "i". Es decir, el riesgo en general puede entenderse como la probabilidad de pérdida durante un período de tiempo " $\mathrm{t}$ " dado.

En el caso particular, el presente investigador, así como el personal de la mayoría de los entes relacionados con las actividades formativas en el ámbito universitario nacional, asumen la siguiente formula, como más adecuada para la labor señalada:

$$
\mathbf{R}=\mathbf{A} \cdot \mathbf{V}
$$

Es decir, el riesgo es igual a la amenaza multiplicada por la vulnerabilidad existente. Por lo que al minimizar los factores que representan la amenaza o la vulnerabilidad, este tiende a cero.

\section{Aspectos Metodológicos}

La metodología aplicada se corresponde con el enfoque del paradigma cuantitativo, o de naturaleza positivista, el cual según Hurtado y Toro (1998, p.36), trata de “...describir objetivamente el mundo material sin referencia alguna al sujeto observador...". Esto, por cuanto se intentó evaluar las percepciones de los discentes cursantes de la Unidad de Formación "Gestión de Riesgos y Protección Civil”, correspondiente al Trayecto Inicial del Programa Nacional de Formación en Administración (PNFA), de la Universidad Politécnica Territorial del Estado Trujillo "Mario Briceño Iragorry" en su núcleo La Beatriz, por ser el actual investigador el encargado de brindar la formación en el área estudiada durante los últimos años en el mencionado núcleo, no siendo limitante este hecho para que la misma pueda ser extrapolada a otros núcleos o Universidades Territoriales, en los cuales se imparta la misma, o similar, unidad curricular.

De acuerdo a las características del estudio, se utilizó el diseño de campo, que según León y Montero (2010, p.28), "es aquel que se caracteriza por estudiar los problemas que surgen de la realidad y la información requerida debe obtenerse directamente de ella”.

Volumen 23, $N^{\circ} 3$, Septiembre-Diciembre 2019. ISSN 2244-7296 
RIESGOS SOCIONATURALES Y TECNOLÓGICOS EN LAS COMUNIDADES

DESDE LA PERCEPCIÓN DE LOS DISCENTES UNIVERSITARIOS

José Javier Leal Rivero

(pp.32-56)

Cabe resaltar que este diseño, como base diagnostica en los discentes, se llevó a cabo con un carácter explorativo, en las etapas tempranas al inicio de su unidad curricular, señalada ésta previamente. Esto debido a la profundidad y complejidad que amerita el estudio de las percepciones, en el contexto de la sociología y psicología social, para poder cumplir así con los preceptos del método científico y la fenomenología bajo estudio. A los efectos prácticos, para la presente investigación se utilizó un cuestionario con diez (10) ítems, utilizando posibilidades de respuestas cerradas, a modo de selección, siendo el mismo aplicado a setenta (70) estudiantes seleccionados para ser parte de la muestra, quienes fueron seleccionados de manera intencional.

\section{Validez}

La validez del instrumento está referida "al grado en que se mide la concordancia y coherencia que debe tener cada ítems con la variable respectiva del estudio" (Hernández, Fernández y Baptista, 2010, p.200). Por lo tanto, para validar el instrumento aplicado a la las personas conformantes de la muestra, el mismo se sometió a un análisis estadístico que permitió evaluar su validez en relación al constructo que posee. Se obvió, la utilización bastante arraigada de, llevar a cabo una revisión y juicio por parte de expertos, debido a que es difícil acceder a profesionales, al menos en el ámbito local, que manejen de manera suficiente el tema de la Gestión Integral de Riesgos, más allá de los aprendizajes empíricos, y que además cuenten con una base académica sólida que les permita una perspectiva cognitiva y metodológica como la requerida por el enfoque del presente trabajo.

Entonces, respecto a la validez del constructo, se evaluó mediante un análisis exploratorio de factores, sirviéndose de la herramienta informática ya señalada anteriormente, software SPSSv22, la cual simplifica en gran medida el trabajo realizado, el cual es conocido como: Análisis Factorial.

\section{Análisis Factorial}

Tal como lo reseñan, Abad, García, Gil, Olea, Ponsoda y Revuelta (2004, p.64), “El análisis factorial es una técnica estadística multivariante que sirve para estudiar las 
RIESGOS SOCIONATURALES Y TECNOLÓGICOS EN LAS COMUNIDADES

DESDE LA PERCEPCIÓN DE LOS DISCENTES UNIVERSITARIOS

José Javier Leal Rivero

(pp.32-56)

dimensiones que subyacen a las relaciones entre varias variables". Habitualmente, para esta técnica se toman como datos de partida la matriz de correlaciones entre las "n" variables que interesa analizar. Como información final, suministra una matriz de tamaño " $\mathrm{n} \times \mathrm{p}$ ", denominada matriz factorial rotada. Esta matriz contiene las saturaciones de cada variable en cada una de las "p" dimensiones extraídas, y que son las correlaciones, normalmente de Pearson, entre cada variable y cada dimensión.

El análisis factorial se realiza con dos objetivos claves (ibidem):

1.- Determinar cuál es el número de dimensiones o factores que mide un test y descubrir cuál es el significado de cada una y;

2.- Obtener la puntuación de cada sujeto en cada dimensión.

Normalmente, el número de dimensiones que mide un test es mucho menor que el de ítems. Para descubrir su significado y darles sentido es necesario fijarse en las variables que saturan de forma elevada en cada dimensión. Si un factor explica un porcentaje elevado de la varianza total, eso es síntoma de que las saturaciones de las variables en dicho factor son altas, lo que significa que es una dimensión importante a la hora de describir las relaciones entre las variables originales.

De esta forma, el investigador, aprovechando la potencia de la herramienta informática, antes señalada, se decantó por las consideraciones respecto al uso del SPSS en el cálculo de la validez, realizadas por Aguilar (2011); Díaz, Muñoz y De Vargas, D. (2012), quienes coinciden en que la validez se puede desarrollar por constructo, agrupando los ítems del instrumento probabilísticamente en dimensiones. Así de darse que los dominios se han centrado de manera efectiva en una teoría sólida, la estadística corrobora la idoneidad de las agrupaciones realizadas.

Respecto a la interpretación de los resultados arrojados por el software SPSS, en el caso actual su versión 22, los autores antes señalados precisan que (op.cit): las comunalidades evaluadas ítem a ítem, deben arrojar un valor mayor a 0,4 ; la medida de adecuación KaiserMeyer-Olkin (KMO), debe ser mayor a 0,5; la prueba de Esfericidad de Barlteltt, en su grado

Volumen 23, $N^{\circ} 3$, Septiembre-Diciembre 2019. ISSN 2244-7296 


\section{RIESGOS SOCIONATURALES Y TECNOLÓGICOS EN LAS COMUNIDADES DESDE LA PERCEPCIÓN DE LOS DISCENTES UNIVERSITARIOS José Javier Leal Rivero \\ (pp.32-56)}

de significancia debe ser menor a 0,05 y; de realizarse la "Correlación Dominio Total", que es la diferencia con la confiabilidad que es de "Item Total", asumiéndose para el programa el "Índice de Correlación de Pearson" de las dimensiones, también con todos los ítems pero agrupados en ellas.

Entonces, una vez desarrollado y aplicado el instrumento, se procedió al volcado de los datos en el señalado software, obteniéndose para el mismo que la muestra seleccionada y las teorías que fundamentan la investigación, son adecuadas al instrumento y existe asociación ítem a ítem para el mismo.

Respecto a los resultados, de la aplicación del Índice de Correlación de Pearson, se observó que todos los valores arrojados para los diferentes ítems son bastantes altos, mucho mayores a 0,5, confirmando la validez del instrumento aplicado.

En resumen el instrumento aplicado posee las siguientes características de validez:

1. Comunalidades: entre 0,467 y $0,894>$ a 0,4

2. KMO: de $0,55>$ a 0.5

3. Significancia Barltett: igual a $0,000<$ a 0,05 y;

4. Índice de Pearson: entre 0,667 y 0,885 > al valor aceptado de 0,5

\section{Confiabilidad}

Según Hernández et al. (2010, p.200) "la confiabilidad de un instrumento de medición se refiere al grado en que su aplicación repetida al mismo sujeto u objeto produce los mismos resultados". En el presente estudio, para el cálculo de la confiabilidad se utilizó el Coeficiente Alfa de Cronbach, obteniéndose su valoración a través del programa de estadísticas avanzadas SPSSv.22, el cual una vez aplicado el instrumento y procesados los datos colectados, se determinó que la confiabilidad del mismo se ubica en un valor de 0,986, dotándolo de un alto nivel de confiabilidad.

Volumen 23, $N^{\circ} 3$, Septiembre-Diciembre 2019. ISSN 2244-7296 
RIESGOS SOCIONATURALES Y TECNOLÓGICOS EN LAS COMUNIDADES

DESDE LA PERCEPCIÓN DE LOS DISCENTES UNIVERSITARIOS

José Javier Leal Rivero

(pp.32-56)

\section{Presentación de Hallazgos}

A partir de los datos recopilados, mediante el instrumento aplicado a los discentes participantes en las actividades de aprendizaje significativo y colaborativo, mayoritariamente cursantes de la Unidad de Formación "Gestión de Riesgos y Protección Civil", correspondiente al Trayecto Inicial del Programa Nacional de Formación en Administración (PNFA) núcleo La Beatriz, se obtuvieron los siguientes hallazgos, expuestos a continuación de manera correlacional en concordancia con los aspectos metodológicos asumidos y antes expuestos (Gráfico 2).

La gran mayoría, un 70\%, señaló reconocer el término "Desastre" y como segundo hicieron referencia al relacionado con el ambiente, es decir "Naturales", en un $30 \%$ de los casos. Esto conlleva a inferir que la mayor parte de ellos identifican los riesgos con los desastres que son provocados por los eventos asociados a la naturaleza y sus procesos de equilibrio. En relación con la percepción que poseen de encontrarse viviendo, o conviviendo, en un área expuesta a la amenaza de eventos naturales o tecnológicos, se observa como un $86 \%$ de los mismos inmediatamente asocian encontrarse amenazados por eventos que tienen que ver con los fenómenos naturales, muy acorde con las respuestas anteriormente halladas. Sólo un $14 \%$ de los discentes precisó no saber si se encuentra expuesto a alguna amenaza, lo que indica que tal vez no se encuentra claro con los conceptos relacionados a las mismas.

Para precisar, si los mismos han sido expuestos a alguna situación de desastre, por eventos de tipo naturales, sociales o tecnológicos y que hayan afectado la comunidad donde reside, se observa como $70 \%$ aseguró haber sido afectado por algún evento del tipo socionatural, pudiéndose deber este hecho a que los mismos residan en alguna de las zonas afectadas por las vaguadas, inundaciones o deslaves, que han afectado al estado Trujillo en años recientes.

Sólo $30 \%$ de los encuestados refiere que nunca se ha visto afectado por este tipo de eventos, lo que señala que su área de habitación o desenvolvimiento cotidiano se encuentra en una zona bastante segura, o poco propensa a sufrir los embates de los procesos naturales,

Volumen 23, $N^{\circ} 3$, Septiembre-Diciembre 2019. ISSN 2244-7296 


\section{RIESGOS SOCIONATURALES Y TECNOLÓGICOS EN LAS COMUNIDADES DESDE LA PERCEPCIÓN DE LOS DISCENTES UNIVERSITARIOS José Javier Leal Rivero \\ (pp.32-56)}

especialmente los relacionados con las lluvias, recordando que no se han dado otro tipo de eventos en la región, al menos no a gran escala.

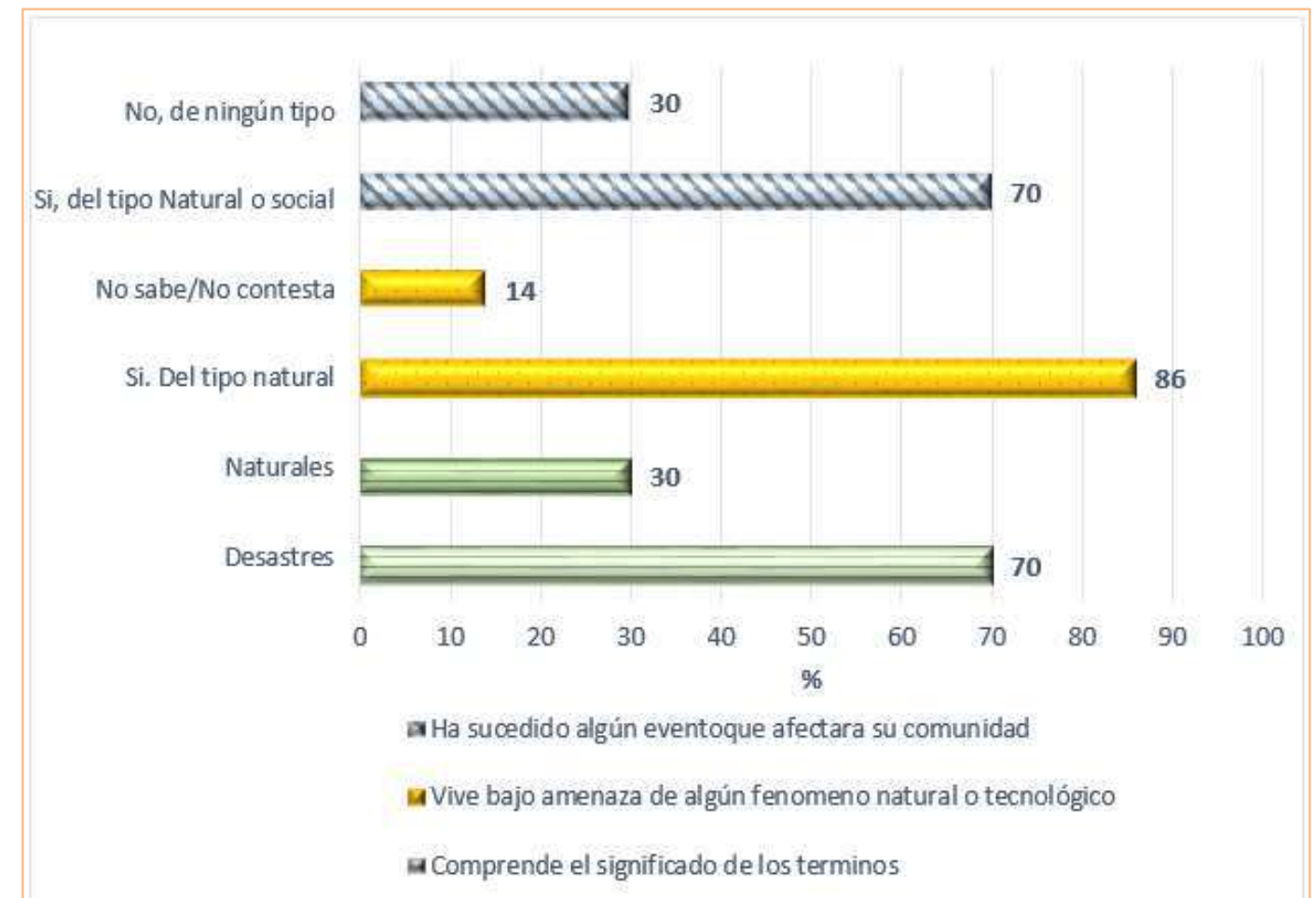

Grafico 2.Identificación de Conocimientos y Saberes, previos respecto a los Riesgos y Desastres. Fuente: Instrumento aplicado. Leal, 2016.

\section{Conclusiones}

En el contexto de la perspectiva social del riesgo, es sin duda la valoración de la percepción colectiva del mismo, una importante herramienta para su Gestión Integral relacionándolo con los fenómenos socionaturales y tecnológicos, requiriéndose entonces siempre desarrollar un análisis integrado de los dos sistemas que lo componen: lo natural o peligrosidad y; el humano conformado por la exposición y la vulnerabilidad. Entendiéndose así que, el enfoque prevaleciente para la forma en que se percibe el riesgo entre las comunidades, y por ende entre los discentes, es el que se refiere a la forma en que un

Volumen 23, $N^{\circ} 3$, Septiembre-Diciembre 2019. ISSN 2244-7296 
RIESGOS SOCIONATURALES Y TECNOLÓGICOS EN LAS COMUNIDADES

DESDE LA PERCEPCIÓN DE LOS DISCENTES UNIVERSITARIOS

José Javier Leal Rivero

(pp.32-56)

individuo interpreta y valora los posibles efectos y peligros de un riesgo de manera directa e inmediata.

Por tanto, la percepción que poseen los discentes de la Universidad Politécnica Territorial del Estado Trujillo "Mario Briceño Iragorry", en relación a los Riesgos Socionaturales y Tecnológicos, que les puedan afectar también a sus comunidades, se encuentra como en la mayoría de la población, canalizada hacia aquellos eventos relacionados con los fenómenos de la naturaleza. Eso limita fuertemente que se amplié el enfoque de los riesgos hacia los aspectos sociales y tecnológicos, disminuyéndose así la capacidad de identificar los factores que se comportan como amenazas y vulnerabilidades, con su respectivo debilitamiento en el esfuerzo para minimizar el riesgo mismo, trayendo como consecuencia un mayor nivel de exposición a las amenazas antrópicas.

\section{Recomendaciones}

Es recomendable retomar el desarrollo del Programa Nacional de Formación en Gestión de Riesgos y Administración de Desastres, tal como fue planteado desde el año 2010, por los profesionales que dedicaron su esfuerzo y compromiso, a partir del conocimiento que a través de los años acumularon como académicos en instituciones tales como el Instituto Universitario de Tecnología de Ejido, hoy en día Universidad Politécnica Territorial de Mérida Kléber Ramírez (UPTM), el Centro de Investigación en Gestión Integral de Riesgos (CIGIR), la Universidad de Los Andes (ULA-Mérida) y el propio investigador por parte del IUTET, actualmente UPTTMBI, así como sus experiencias de campo en la atención de los diferentes eventos y hechos que se convirtieron en desastres asociados con la naturaleza y otros de origen antrópico. Esto debido, al hecho que a pesar de haberse utilizado el trabajo originado por los actores mencionados en la creación del PNF en Protección Civil, el cual le fue asignado exclusivamente a la Universidad Nacional Experimental de la Seguridad (UNES), limitando su alcance poblacional, el mismo se encuentra restringido a la Administración de Desastres, por el enfoque propio de la UNES como ente académico de aspectos relativos a la seguridad del Estado, entendiéndose así, que fue un retroceso en el

Volumen 23, $N^{\circ} 3$, Septiembre-Diciembre 2019. ISSN 2244-7296 


\section{RIESGOS SOCIONATURALES Y TECNOLÓGICOS EN LAS COMUNIDADES \\ DESDE LA PERCEPCIÓN DE LOS DISCENTES UNIVERSITARIOS \\ José Javier Leal Rivero}

(pp.32-56)

cambio de paradigmas que a nivel mundial y Venezuela, se habían planteado de asumir el trabajo en las causas y no en las consecuencias, es decir Prevenir más y Atender menos calamidades.

\section{Referencias}

Abad, J., García, C., Gil, B.,Olea, J.,Ponsoda, V. y Revuelta, J. (2004). Introducción a la Psicometría Teoría Clásica de los Tests y Teoría de la Respuesta al Ítem. Madrid: UNAM.

Aguilar, N (2011). Prueba de Validez. Demo de IBM SPSSPASW 18. Cerro de Pasco, Perú: Universidad Nacional de Daniel Alcides Carrión.

ArtiedaGonzález, J. y Palacios Morera, M. (2000). Procesos de Degradación Ambiental e Imágenes de Satélite: Aplicaciones de Spot. Madrid: Aprensa.

Cardona Arboleda, O. (2001). Estimación Holística del Riesgo Sísmico utilizando Sistemas Dinámicos Complejos. Tesis Doctoral publicada. Universidad Politécnica de Cataluña. Barcelona, España.

Cardona Arboleda, O. (2003) Indicadores para la Medición del Riesgo: Fundamentos para un enfoque metodológico. IADB/IDEA Programa de Indicadores.

Cardona Arboleda, O. (2007). Gestión integral de riesgos y desastres. Barcelona, España: CIMNE

Carreño, M. (2007). A disasterriskmanagement performance index. NatHazards, 41(1), 1-20.

Díaz, L., Muñoz, A. y De Vargas, D. (2012) Confiabilidad y validez del cuestionario de espiritualidad de Parsian yDunning en versión española. Rev. Latino-Am. Enfermagem 20(3), 1-8.

EIRD, (2004). Estrategia Internacional para la Reducción de Desastres (2004). Vivir con el Riesgo extracto del Informe Mundial sobre las iniciativas para la reducción de desastres.EIRD, Ginebra. Suiza. Naciones Unidas.

Hernández Sampieri, R. Fernández Collado, C. y Baptista Lucio, P. (2010) Metodología de la Investigación. 5ta. Ed. México: Mc Graw Hill.

Hurtado León, I, y Toro Garrido, J. (1998). Paradigmas y métodos de investigación en tiempos de cambo. México:McGraw Hill

Leal Rivero, J. (2016). Fortalecimiento de la Cultura en Promoción y Aprendizaje para la Gestión Integral de Riesgos Socionaturales y Tecnológicos en Discentes de la

Volumen 23, N³, Septiembre-Diciembre 2019. ISSN 2244-7296 


\section{RIESGOS SOCIONATURALES Y TECNOLÓGICOS EN LAS COMUNIDADES DESDE LA PERCEPCIÓN DE LOS DISCENTES UNIVERSITARIOS José Javier Leal Rivero}

UPTTMBI. Trabajo de Ascenso a la Categoría de Asociado no publicado. Universidad Politécnica Territorial del Estado Trujillo "Mario Briceño Iragorry”. Valera

León García, O. y Montero García, I. (2010).Diseño de investigaciones: Introducción a la lógica de la investigación en Psicología y Educación. Madrid: McGraw Hill

Ley de Gestión Integral de Riesgos Socionaturales y Tecnológicos (2009). Publicada en GacetaOficial No 39.095. Caracas. Venezuela

Mansor, A. (2004). Spatial Technology for natural risk management. Disaster Prevention and Management, 13(5), 364-373.

Myers, A., (2004) Preparing communities for disasters: issues and processes for government readiness. Disaster Prevention and Management, 13(2), 140-152.

Perry, R. (2003). Emergency Operations Centres in an Era of Terrorism: Policy and Management Functions. Journal of Contingencies and Crisis Management, 11(4).151159.

Perry Ronald, W. \&Quarantelli, E L. (2004). What is a Disaster? New Answers to Old Questions.Philadelphia: Xlibris Corporation.

Shaluf, I. y Fakhrul-Razi, A. (2013). A review of disaster and crisis. DisasterPrevention and Management, (12)1,24-32.

UNISDR (2015). El GAR de bolsillo 2015. Hacia el desarrollo sostenible: El futuro de la gestión del riesgo de desastres. Ginebra, Suiza: Organización de Naciones Unidas.

Volumen 23, N³, Septiembre-Diciembre 2019. ISSN 2244-7296 